\title{
Frequency-dependent gating of synaptic transmission and plasticity by dopamine
}

\author{
Hiroshi T. Ito ${ }^{1}$ and Erin M. Schuman ${ }^{2, *}$ \\ 1. Division of Biology, California Institute of Technology, USA \\ 2. Division of Biology, California Institute of Technology and Howard Hughes Medical Institute, USA \\ Edited by: Rafael Yuste, Columbia University, New York City, USA \\ Reviewed by: Gabor Tamas, University of Szeged, Hungary
}

The neurotransmitter dopamine (DA) plays an important role in learning by enhancing the saliency of behaviorally relevant stimuli. How this stimulus selection is achieved on the cellular level, however, is not known. Here, in recordings from hippocampal slices, we show that DA acts specifically at the direct cortical input to hippocampal area CA1 (the temporoammonic (TA) pathway) to filter the excitatory drive onto pyramidal neurons based on the input frequency. During low-frequency patterns of stimulation, DA depressed excitatory TA inputs to both CA1 pyramidal neurons and local inhibitory GABAergic interneurons via presynaptic inhibition. In contrast, during high-frequency patterns of stimulation, DA potently facilitated the TA excitatory drive onto CA1 pyramidal neurons, owing to diminished feedforward inhibition. Analysis of DA's effects over a broad range of stimulus frequencies indicates that it acts as a high-pass filter, augmenting the response to high-frequency inputs while diminishing the impact of low-frequency inputs. These modulatory effects of DA exert a profound influence on activity-dependent forms of synaptic plasticity at both TA-CA1 and Schaffer-collateral (SC)-CA1 synapses. Taken together, our data demonstrate that DA acts as a gate on the direct cortical input to the hippocampus, modulating information flow and synaptic plasticity in a frequency-dependent manner.

Keywords: hippocampus, area CA1, dopamine, temporoammonic pathway, frequency-dependent filter, LTP, LTD

\section{INTRODUCTION}

Midbrain dopaminergic neurons increase their firing activity when animals receive unexpected rewards or experience a novel environment-both the hedonic value and familiarity of stimuli are important determinants in learning (Horvitz, 2000; Schultz and Dickinson, 2000). The learningrelated activities of dopaminergic neurons may induce modifications of the neural networks that underlie behavioral plasticity (McClure et al., 2003; Montague et al., 2004).

One of the targets of dopaminergic neurons is the hippocampus, a brain structure crucial for some types of learning and memory (Gasbarri et al., 1994a, 1994b, 1996a; Scoville and Milner, 1957; Squire et al., 2004; Swanson, 1982; Zola-Morgan and Squire, 1986). Dopamine (DA) is released in the hippocampus when animals are exposed to nove environments (Ihalainen et al., 1999), influencing hippocampal-dependent learning (Gasbarri et al., 1996b).

In the hippocampus, the primary targets of dopaminergic neurons are the subiculum and area CA1 (Gasbarri et al., 1997). CA1 pyramidal neurons receive two distinct excitatory synaptic inputs: one from area CA3 (the Schaffer-collateral (SC) pathway), and the other from the entorhinal cortex (the temporoammonic (TA) pathway) (Cajal, 1911). Each pathway appears to have a distinct function in learning (Brun et al., 2002; Remondes and Schuman, 2004; Steffenach et al., 2002) and may be differentially

\footnotetext{
* Correspondence: Erin M. Schuman, Division of Biology, California Institute of Technology and Howard Hughes Medical Institute, 1200 E. California Blvd, Pasadena, CA 91125, USA. e-mail: schumane@caltech.edu

Received: 25 Jun. 2007; paper pending published: 17 Sep. 2007; accepted: 15 0ct. 2007; published online: 02 Nov. 2007

Full citation: Frontiers in Neural Circuits (2007) 1:1 doi: 10.3389/neuro.04/001.2007 Copyright: (๑) 2007 Ito, Schuman. This is an open-access article subject to an exclusive license agreement between the authors and the Frontiers Research Foundation, which permits unrestricted use, distribution, and reproduction in any medium, provided the original authors and source are credited.
}

modulated depending on the familiarity of the environment (Hasselmo and Schnell, 1994; Otmakhova and Lisman, 1999; Otmakhova et al., 2005). This differential pathway modulation may contribute to the distinct informational representation between area CA1 and CA3, as observed in in vivo animals (Lee et al., 2004; Leutgeb et al., 2004; Vazdarjanova and Guzowski, 2004). Indeed, independent modulation of the two pathways has been hypothesized to play a significant role in learning (Guzowski et al., 2004; Hasselmo et al., 1996; Knierim et al., 2006; Lisman and Otmakhova, 2001). Here we explored how DA modulates the signal integration of these two hippocampal pathways with the goal of understanding how DA might regulate information selection during learning.

\section{MATERIALS AND METHODS}

\section{Hippocampal slice preparation}

Slices were prepared from 25 to 35 day-old Sprague-Dawley rats (Harlan) and microdissected to isolate the TA pathway, as described previously (Dvorak-Carbone and Schuman, 1999a). In brief, a vibrating microtome (EMS 0TS4000 or Leica VT1000S) or a tissue chopper (Stoelting) was used to cut hippocampal slices (500 $\mu \mathrm{m}$ thickness, except $300 \mu \mathrm{m}$ for Figures $1 \mathrm{C}$ and $1 \mathrm{D}$ ) in ice-cold oxygenated artificial cerebrospinal fluid (ACSF) containing (in mM) $119 \mathrm{NaCl}, 2.5 \mathrm{KCl}, 1.3 \mathrm{MgSO}_{4}, 2.5 \mathrm{CaCl}, 1.0$ $\mathrm{NaH}_{2} \mathrm{PO}_{4}, 26.2 \mathrm{NaHCO}_{3}, 11.0$ glucose. Slices were recovered at room temperature for at least 1 hour in an interface chamber, and transferred to a submerged recording chamber perfused with ACSF at $24.5-25.5^{\circ} \mathrm{C}$. The dentate gyrus and CA3 were removed to eliminate the possible activation of the trisynaptic pathway or perforant path projection to area CA3. Concentric bipolar tungsten electrodes $(\mathrm{FHC})$ and stimulus isolators (Axon Instruments) were used for the stimulation.

\section{Electrophysiology}

Extracellular field potential recordings were made with 1-3 $\mathrm{M} \Omega$ resistance microelectrodes filled with $3 \mathrm{M} \mathrm{NaCl}$ using a bridge amplifier (Axoclamp 

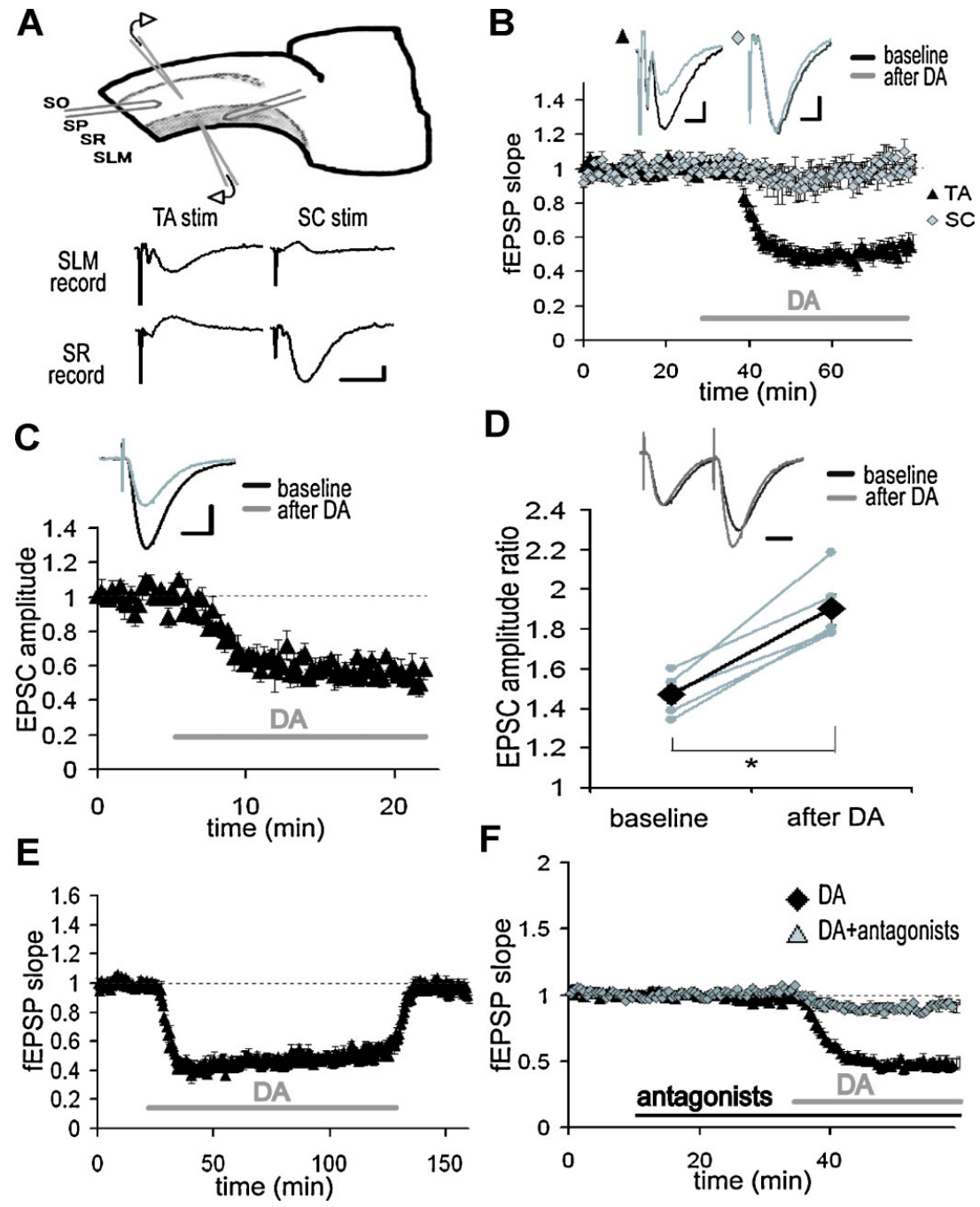

Figure 1. Inhibition of TA-CA1 pyramidal excitatory synaptic transmission by DA. (A) Simultaneous extracellular field recording from SC-CA1 and TA-CA1 synapses. Extracellular recording and stimulating electrodes are placed in SLM or SR, respectively. SO, stratum oriens; SP, stratum pyramidale. Shown are representative fEPSP recorded in SLM or SR as a result of TA or SC stimulation. Scale bar $=0.1 \mathrm{mV}, 10 \mathrm{~ms}$. (B) Application of DA (20 $\mu$ M; indicated by bar) significantly depressed the fEPSP slope of TA-CA1 synapses, but did not affect SC-CA1 synapses $(n=6)$. Scale bar $=0.1 \mathrm{mV}, 5 \mathrm{~ms}$. (C) Whole-cell patch-clamp recording from CA1 pyramidal neurons in the presence of the GABA $A_{A \text { and } B}$ receptor antagonists, bicuculline $(10 \mu \mathrm{M})$ and CGP $55845 A(1 \mu \mathrm{M})$. After DA application, the EPSC evoked by TA pathway stimulation was significantly depressed, $p<0.01(n=4)$. Scale bar $=50 \mathrm{pA}, 20 \mathrm{~ms}$. (D) Paired-pulse facilitation analysis using the same condition described in C. Pulse interval was $50 \mathrm{~ms}$. Data from individual experiments are represented by small gray circles; large diamonds represent the mean. After DA application, paired-pulse facilitation of the TA-CA1 EPSC was significantly enhanced, $p<0.01(n=5)$. Scale bar $=20 \mathrm{~ms}$. (E) Recovery from long-term application of DA ( $n=4)$. (F) Blockade of DA-induced depression at TA-CA1 synapses by DA receptor antagonists, SKF $83566(1 \mu M)$ and Sulpiride $(10 \mu M)(D A: n=8, D A$ antagonists: $n=5)$.

2B, Axon Instruments). Whole-cell voltage-clamp recordings from CA1 pyramidal neurons were made without visualization with an Axopatch 1D or 200B (Axon Instruments). Internal solution of patch pipettes was (in $\mathrm{mM}) 115$ potassium gluconate, $20 \mathrm{KCl}, 10$ sodium phosphocreatine, 10 HEPES, 2 MgATP, 0.3 NaGTP (pH 7.3). In addition, to minimize possible postsynaptic current modulation by DA, pipette solutions contained (in $\mathrm{mM}) 5$ QX314, 10 TEA, 1 4AP. Membrane voltage was clamped at $-60 \mathrm{mV}$ (without liquid junctional potential correction, absolute value of holding current $<100 \mathrm{pA}$ in all recordings). Membrane capacitance was cancelled and series resistance was compensated (70-80\%). Recordings were discarded when the series resistance was over $20 \mathrm{M} \Omega$ or either series or membrane resistance changed more than $20 \%$ during data acquisition. For sharp-electrode intracellular recordings from CA1 interneurons, electrodes were blindly advanced at the border of stratum radiatum (SR) and stratum lacunosum-moleculare (SLM) and neurons were penetrated with a short buzz with an Axoclamp $2 B$ amplifier. Interneurons were identified by both spike activities and morphology using biocytin staining after the recording. Sharp electrodes were $90-150 \mathrm{M} \Omega$ in resistance and contained $2 \mathrm{M}$ potassium acetate. No tonic current was injected during recordings. Input resistance and membrane potential were monitored and recordings were discarded when either of them changed more than $20 \%$ during data acquisition. In our recordings from interneurons $(n=5)$, average input resistance: $45.2 \pm 7.8 \mathrm{M} \Omega$ and average membrane potential: $-66.6 \pm 3.0 \mathrm{mV}$. In the experiments using low $\left[\mathrm{Ca}^{2+}\right]_{\text {ext }}$, extracellular calcium concentration was $1.25 \mathrm{mM}$, instead $2.5 \mathrm{mM}$, and the total divalent cation was compensated by adding magnesium. For frequency-dependent modulation analysis, 100 pulses stimulation at the indicated frequencies were applied after 
baseline responses were stable for at least 10 minute. The following stimulation protocols were used: for long-term potentiation (LTP) induction, $100 \mathrm{~Hz} 100$ pulses, repeated 4 times at 5 minute intervals; for longterm depression (LTD) induction, $1 \mathrm{~Hz} 900$ pulses. For joint theta-burst stimulation (TBS) application, 10 bursts of either 5 (SC) or 10 (TA) at $100 \mathrm{~Hz}$ with $200 \mathrm{~ms}$ interburst interval was repeated twice at 30 second interval (the onset of TA TBS precedes SC TBS by $20 \mathrm{~ms}$ ). All stimulus pulses were of the same length and amplitude as test pulses. Test pulses were applied once every 30 second for extracellular field recordings, every 15 second for whole-cell recordings and every 10 second for sharp electrode intracellular recordings. Drugs were applied by dilution of concentrated stock solutions into the perfusion medium. The final concentration of bath-applied DA was $20 \mu \mathrm{M}$ (e.g., Otmakhova and Lisman, 1999). In acute DA application experiments, $500 \mu \mathrm{L}$ of ACSFdiluted DA $(60 \mu \mathrm{M})$ was directly applied into the recording chamber. The volume of recording chamber is $\sim 1 \mathrm{~mL}$, thus estimated final concentration of DA is $\sim 20 \mu \mathrm{M}$. The flow rate of ACSF was approximately $1.5 \mathrm{~mL} /$ minute.

\section{Data analysis}

Data were collected by a custom program using the LabView data acquisition system (National Instruments) for extracellular recordings, or DigiData 1200 and pClamp 9 (Axon Instruments) for intracellular recordings. All numerical values listed represent mean \pm SEM. Depression and potentiation were measured at $30-35$ and 50-60 minute after plasticity induction, respectively, relative to baseline (average of the slopes of the field EPSPs (fEPSPs) for 15 minute prior to plasticity induction). For plasticity experiments under DA application, the baseline fEPSP was normalized to the depressed state. For analysis of the waveforms during 100 pulses stimulation, stimulation artifacts (and fiber volleys in field recordings) were excluded and the gaps were linearly connected, and the last excitatory potential or current (100th stimulus response) was measured by a custom program in Matlab (MathWorks). Steady-state potentials or currents were measured to exclude the influence of initial states of the network at the beginning of stimulation. Wilcoxon rank sum test was performed for all statistical analysis except Figure 9E, for which an ANOVA was performed.
A

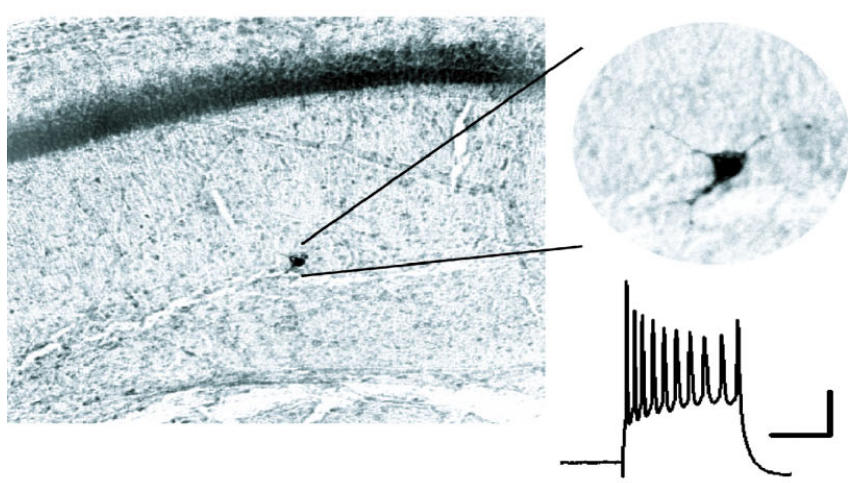

C

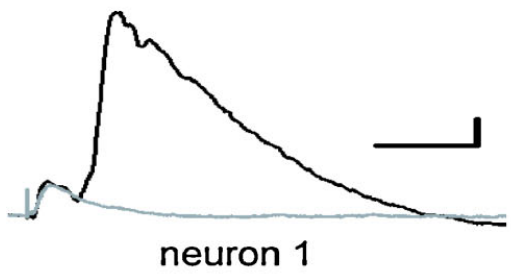

D

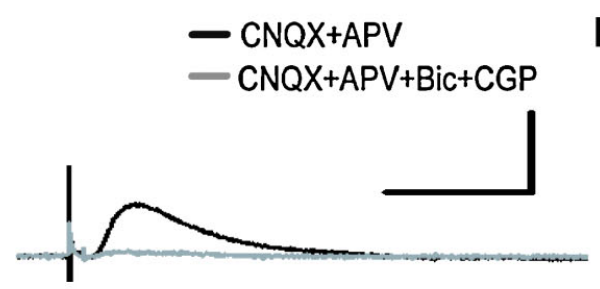

B

E
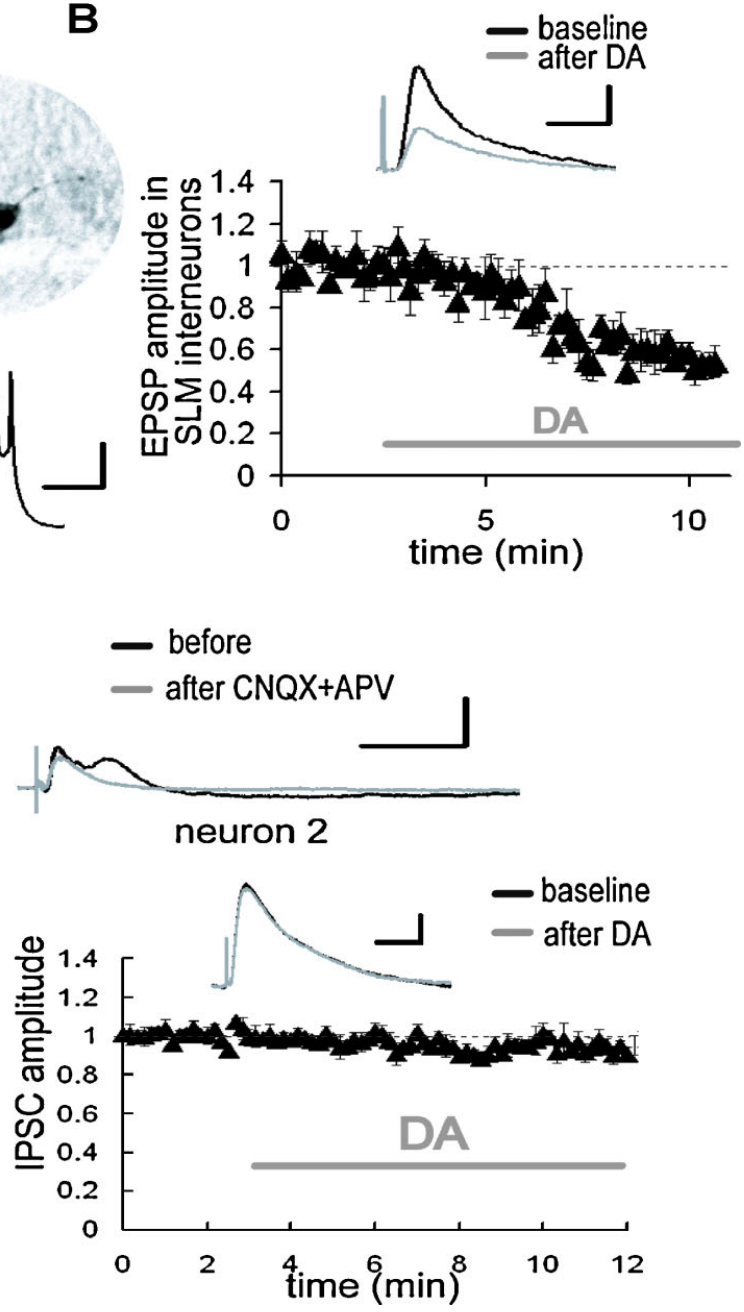

Figure 2. DA-induced depression of excitatory inputs to SLM interneurons. (A) Intracellular recording from SLM interneurons. Biocytin-filled electrodes were used for the staining of SLM interneurons. The inset shows representative spike activities following current injection. Scale bar $=50 \mathrm{~ms}, 20 \mathrm{mV}$. (B) After DA application, the EPSP evoked by the TA pathway stimulation was significantly depressed. $p<0.01(n=5)$. Scale bar $=1 \mathrm{mV}, 20 \mathrm{~ms}$. (C) Whole-cell voltage-clamp recordings from CA1 pyramidal neurons at a holding potential of $0 \mathrm{mV}$. The late component of the IPSC disappeared after excitatory blockade with CNQX (10 $\mu$ M) and APV $(25 \mu \mathrm{M})$. The size of late IPSC showed large variability among recorded neurons. Scale bar $=50 \mathrm{pA}, 100 \mathrm{~ms}$. (D) The monosynaptic IPSC was blocked by GABA receptor antagonists, bicuculline $(10 \mu \mathrm{M})$ and CGP 55845A $(1 \mu \mathrm{M})$. Scale bar $=50 \mathrm{pA}, 100 \mathrm{~ms}$. (E) DA did not influence monosynaptic IPSC. Scale bar $=20 \mathrm{pA}, 50 \mathrm{~ms}$. 


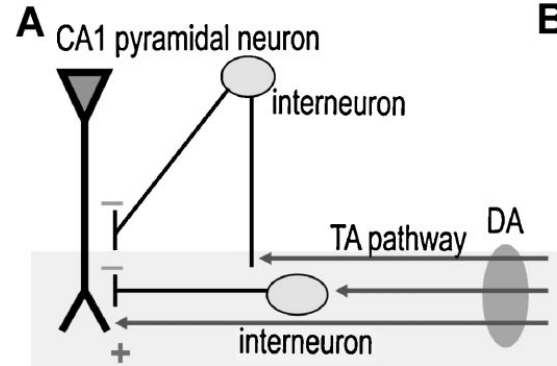

C
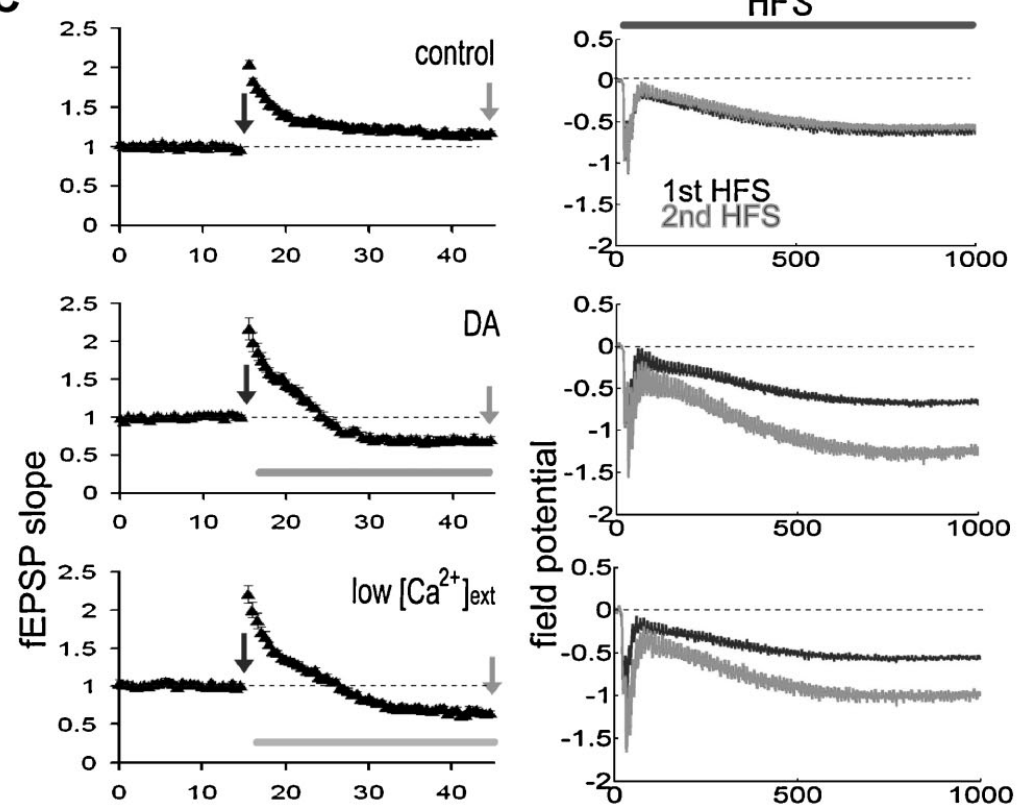

.
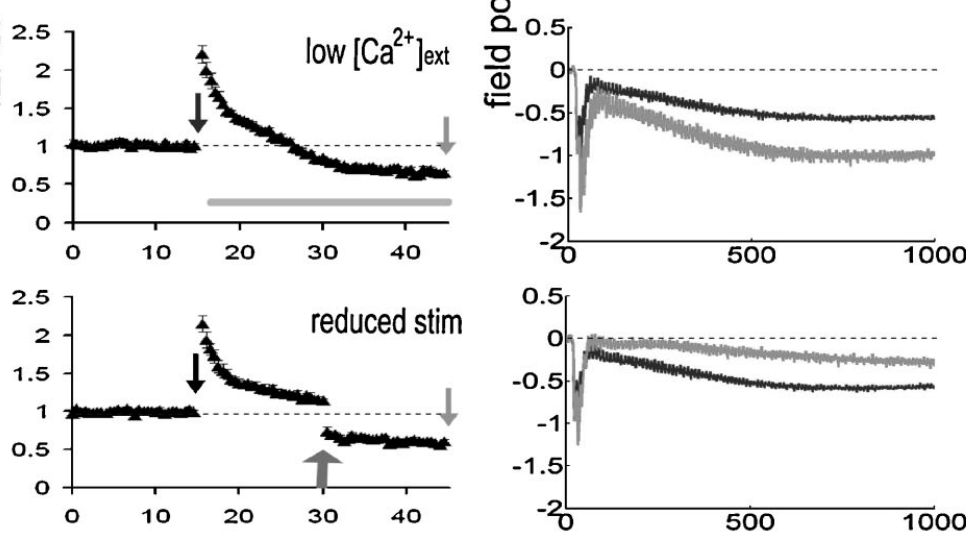

D

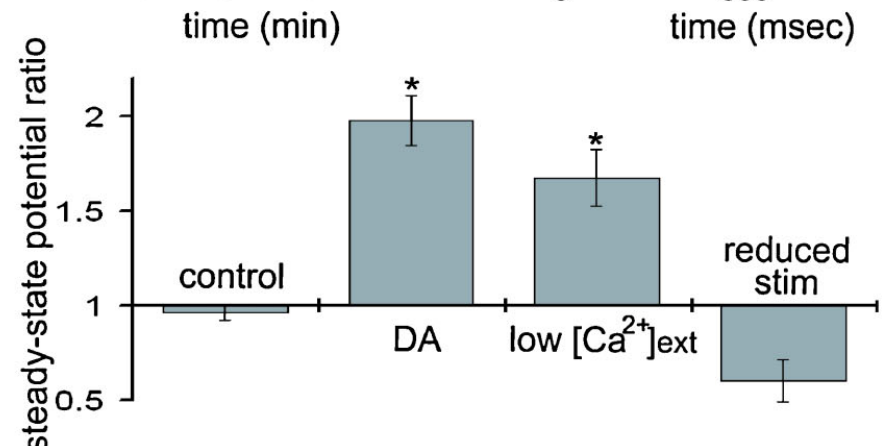

Low frequency stim

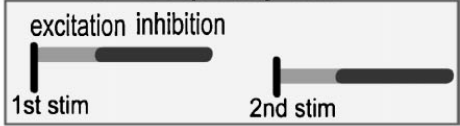

High frequency stim

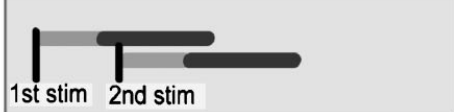

HFS
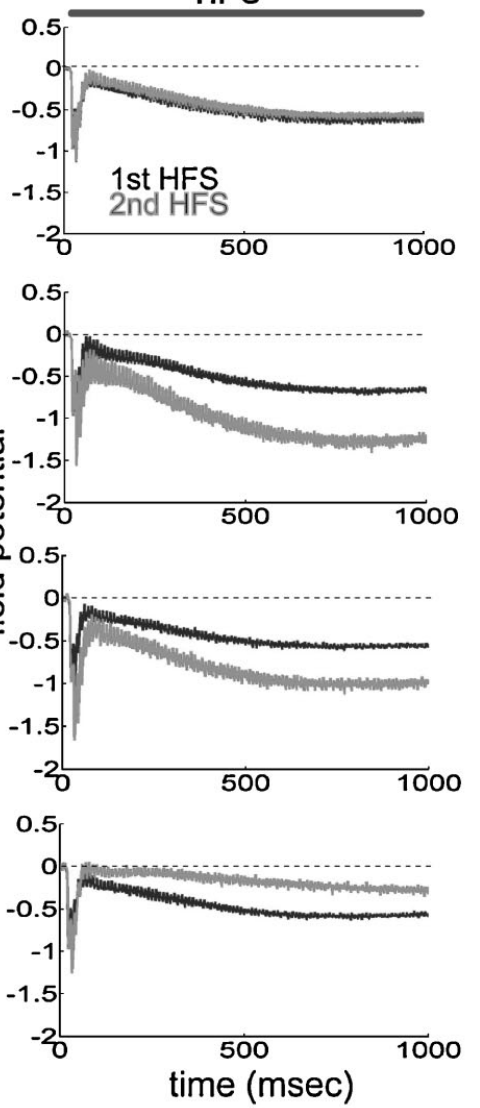

Whe steady-state field potential induced by HFS. (A) Scheme of the TA-CAT synapse. The with both pyramidal neurons and interneurons. Interneurons in turn inhibit pyramidal neurons (feedforward inhibition). Depression of excitatory inputs onto interneurons by DA reduces inhibition of pyramidal neurons. (B) Frequency-dependent effect of feedforward inhibition. In contrast to the direct excitatory TA-CA1 input, the TA pathway exerts a disynaptic modulation of inhibition. Thus, the inhibition of pyramidal neurons after TA stimulation is delayed, relative to the excitation. In pyramidal neurons, the TA pathway-evoked inhibition does not affect excitation during low-frequency stimulation, because of the delay in inhibition. However, during HFS, inhibition can effectively suppress subsequent excitatory responses. (C) Extracellular field recording from TA-CA1 synapses. Left, HFS was applied at 15 and 45 minute (indicated by arrows). Field potential traces during HFS are normalized to the baseline fEPSP amplitude prior to HFS application. The field potential at the end of the HFS (100th stimulus response) was measured (steady-state potential). Right, the average waveforms during HFS are shown. DA: after first HFS, DA was applied (indicated by bar). Low [Ca $\left.{ }^{2+}\right]_{\text {ext }}$ : after first HFS, the extracellular calcium concentration was reduced from 2.5 to $1.25 \mathrm{mM}$ (indicated by bar). Reduced stimulus: at 30 minute, the stimulation current was reduced to produce a small fEPSP comparable to that observed during DA application (indicated by arrow) ( $n=5$ for each group). (D) Ratio of steady-state potentials observed during second and first HFS epochs. DA application induced a significantly $(p<0.01)$ larger steady-state potential that was also mimicked by reduction of $\left[\mathrm{Ca}^{2+}\right]_{\text {ext }}$. 
A

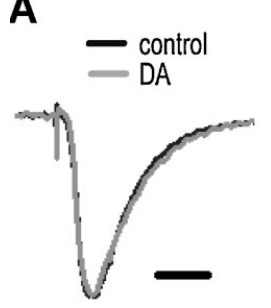

B
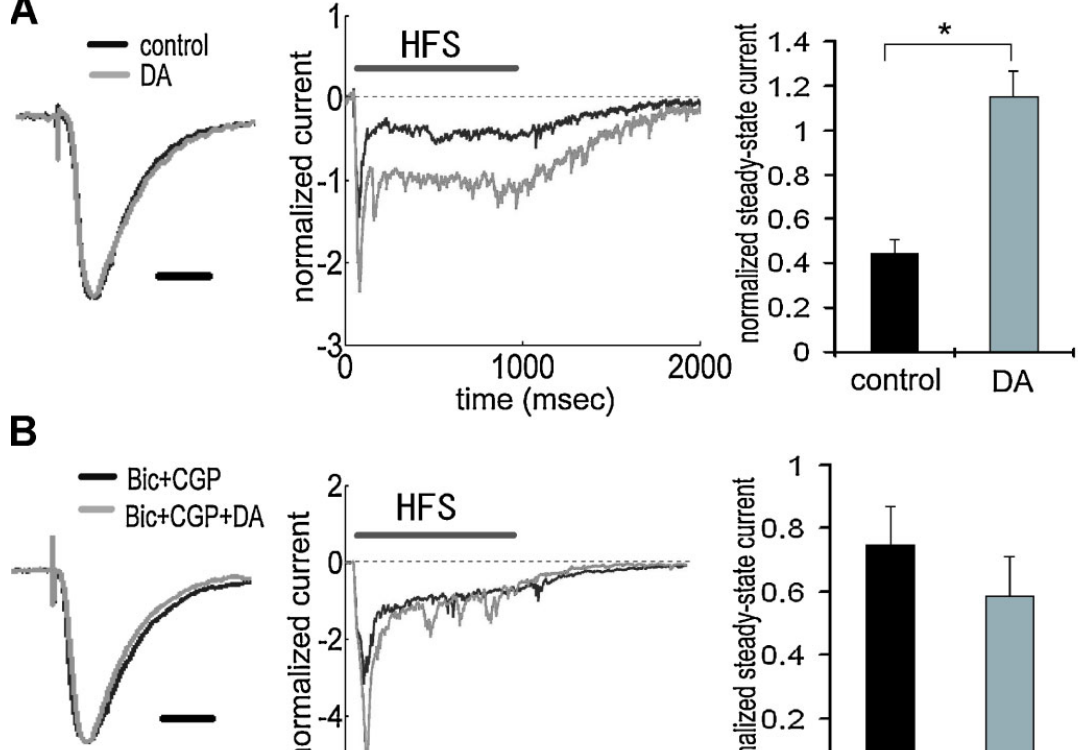
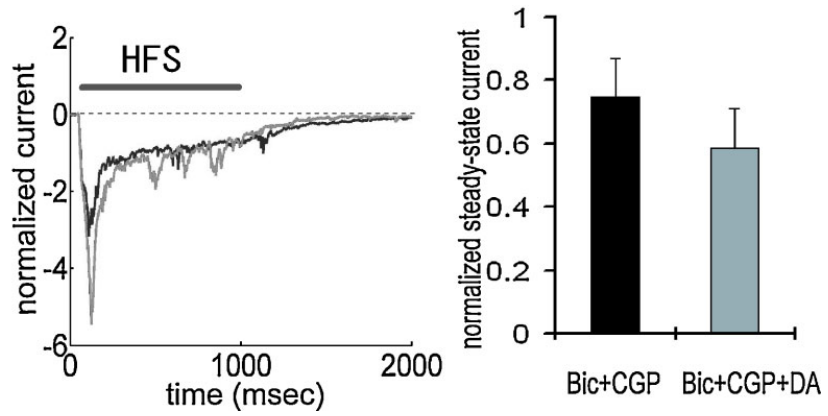

Figure 4. Enhancement of TA-CA1 synaptic efficacy during HFS via DA-induced disinhibition. (A) Whole-cell voltage-clamp recording from CA1 pyramidal neurons. Waveforms represent the average of all data, showing normalized baseline EPSC (left) and current during HFS (middle). There was no difference in the kinetics of EPSC waveforms obtained with or without DA application. Waveforms during HFS (100 Hz 100 pulses) were normalized to baseline EPSC amplitude prior to HFS, and the current at the end of HFS (100th stimulus response) was measured (steady-state current). The right figure shows the analysis of steady-state current, showing that $D A$ induced a significantly $(p<0.01)$ larger steady-state current. Input resistances were not significantly different (in $M \Omega$ ); control: $85.8 \pm 9.3, D A: 85.0 \pm 9.0$ ( $n=6$ for each group). Scale bar $=20 \mathrm{~ms}$. (B) Same experimental procedure as $\boldsymbol{A}$ under GABA receptor blockade by bicuculline and CGP 55845A. GABA blockade attenuated the enhancement of steady-state current by DA. Input resistances were not significantly different (in $M \Omega) ; B i c+C G P: 102.0 \pm 7.9, B i c+C G P+D A: 98.0 \pm 4.6(n=5$ for each group). Scale bar $=20 \mathrm{~ms}$.

\section{RESULTS \\ DA selectively depresses excitatory synaptic transmission at TA-CA1 pyramidal neuron synapses}

To examine the differential influence of DA on the two excitatory inputs to area CA1, we made extracellular field recordings from both the SC pathway and the TA pathway in hippocampal slices (Figure 1A). As previously described (Otmakhova and Lisman, 1999), when DA (20 $\mu$ M) was applied to the bathing solution, the fEPSP evoked by the TA pathway stimulation was depressed, whereas the fEPSP by the SC pathway stimulation was not significantly altered (Figure 1B; DA: $49.2 \pm 8.8 \%$, SC: $93.6 \pm 15.8 \%$, mean percent of baseline 20-30 minute after DA application). To identify the synaptic locus of DA's effect, we conducted whole-cell voltageclamp recordings from CA1 pyramidal neurons. DA also depressed the EPSC evoked by the TA pathway stimulation (Figure 1C; $56.8 \pm 2.3 \%$, average of 15-20 minute after DA application), indicating a decrease in excitatory neurotransmission. We also analyzed paired-pulse facilitation [inversely correlated with vesicle release probability (Dobrunz and Stevens, 1997; Katz and Miledi, 1968; Zucker, 1973)], before and after DA application. After DA application, paired-pulse facilitation was significantly enhanced (Figure 1D), suggesting that DA acts, at least in part, via an inhibition of neurotransmitter release. The DA-induced depression was reversible (Figure 1E) and blocked by DA receptor antagonists (Figure 1F).

\section{DA depresses excitatory synaptic transmission at TA-interneuronal synapses}

In addition to excitatory connections with CA1 pyramidal neuron dendrites, the axons of the TA pathway also make synapses with interneurons in area CA1 (Freund and Buzsaki, 1996). Among the various classes of interneurons present, the interneurons located at the border between SR and SLM receive excitatory synapses from the TA pathway (DvorakCarbone and Schuman, 1999b; Lacaille and Schwartzkroin, 1988). We obtained intracellular recordings from those interneurons and examined the effects of DA on the TA-interneuron excitatory synapse. We found that DA also depressed the TA pathway-evoked EPSP in interneurons (Figure 2B; $57.7 \pm 4.2 \%$ in EPSP amplitude, $44.0 \pm 12.5 \%$ in EPSP slope after DA application). Thus, DA depressed the excitatory synaptic inputs at both TA-pyramidal and TA-interneuron synapses. Although we did not examine connections between TA axons and other type of interneurons in area CA1, considering the presynaptic action of DA (Figure 1D), it is possible that other TA-interneuron synapses will be similarly depressed.

Previous studies have reported that relatively strong inhibitory responses can be observed in CA1 pyramidal neurons following TA pathway stimulation (Empson and Heinemann, 1995). Since the TACA1 synapses are primarily excitatory (Desmond etal., 1994), the inhibition of pyramidal neurons is caused by interneurons which receive excitatory inputs from the TA pathway and, in turn, make inhibitory connections with pyramidal neurons (Lacaille and Schwartzkroin, 1988). We thus examined the inhibitory responses in CA1 pyramidal neurons evoked by TA pathway stimulation. Whole-cell monosynaptic IPSCs were recorded from CA1 pyramidal neurons at a holding potential of $0 \mathrm{mV}$ in the presence of glutamate receptor antagonists (CNQX + APV). The monosynaptic IPSC (Figure 2D) was not modulated by DA (Figure 2E), suggesting that DA does not influence inhibitory synaptic transmission. 
A
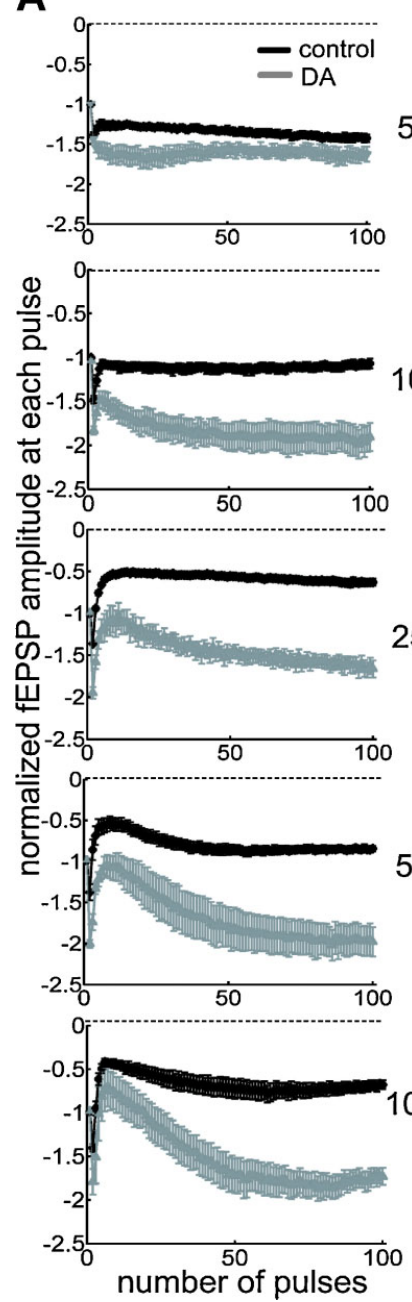

B

$5 \mathrm{~Hz}$

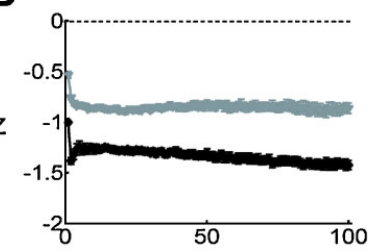

$10 \mathrm{~Hz}$
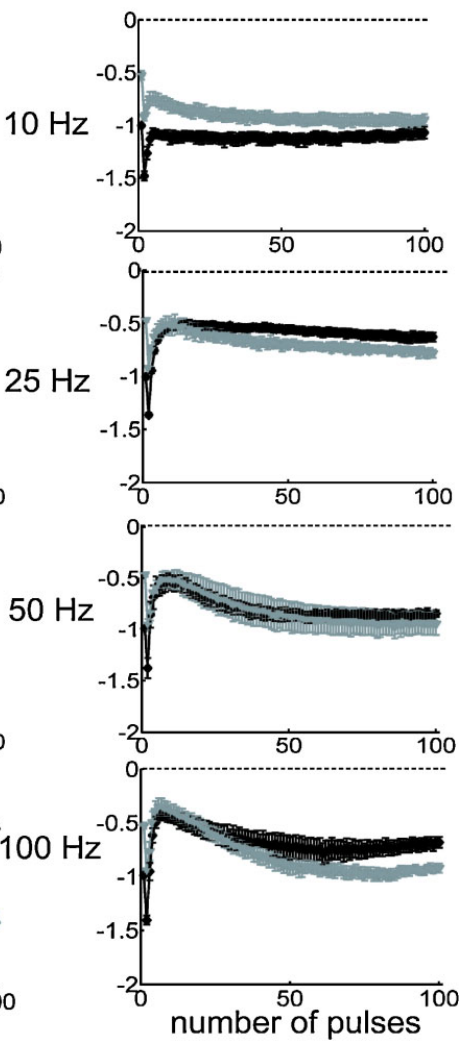

Figure 5. High-pass filtering of TA-CA1 synaptic efficacy by DA. (A) Examination of frequency-dependent modulation by $D A$, using extracellular field recordings. Pulses (100) of different stimulation frequencies, as indicated, were applied. Data were normalized to the baseline fEPSP amplitude prior to stimulation and each mean IEPSP amplitude during 100 pulse stimulation was plotted (from top to bottom; control: $n=5,5,7,6$, and 5, DA: $n=5,5,5,5$, and 5). (B) Same data as in $\boldsymbol{A}$, but normalized to the baseline fEPSP amplitude before, instead of after, DA application.

\section{DA-induced presynaptic inhibition enhances synaptic transmission at TA-CA1 synapses during high-frequency stimulation (HFS)}

The above indicate that DA appears to selectively inhibit the excitatory TA connections with both local interneurons and CA1 pyramidal neurons (Figure $3 A$ ). The DA-induced depression of excitatory inputs to interneurons is predicted to reduce the impact of inhibitory transmission on CA1 pyramidal neurons (disinhibition). Because this inhibitory transmission is disynaptic, the inhibition of pyramidal neurons is delayed relative to excitation. This raises the possibility that DA's ability to modulate the output of the TA-CA1 circuit may be modulated by stimulation frequency. In particular, we predicted that appropriately timed TA stimuli would increase the impact of disinhibition (Figure 3B).

To test this idea, we examined the net effect of DA during epochs of HFS. We made extracellular field recordings from the TA-
CA1 synapses, before, during and after two epochs of HFS $(100 \mathrm{~Hz}$ 100 pulses); the second epoch was delivered after DA application (Figure 3C). To quantify the differences between first and second HFS, we measured the field potential evoked by the last (100th) stimulus (referred to as the "steady-state potential"). Under control conditions, the steady-state potentials observed at the first and second HFS were almost identical (second/first steady-state potential ratio: $0.96 \pm 0.04$ ) (Figure 3D). DA application, however, significantly enhanced the second steady-state potential (ratio: $1.98 \pm 0.13$ ). To examine whether presynaptic inhibition is sufficient to induce this phenomenon, we lowered the extracellular calcium concentration after first HFS (low $\left[\mathrm{Ca}^{2+}\right]_{\text {ext }}$; Figure $3 \mathrm{C}$ ), reducing the probability of neurotransmitter release. This manipulation mimicked the effect of DA, resulting in a larger steadystate potential at the second HFS (ratio: $1.67 \pm 0.15$; Figure 3D). To exclude the possibility that a smaller fEPSP (induced by DA) may itself induce a larger steady-state potential, independent of release probability, we reduced the stimulus strength to imitate the small fEPSP depressed by DA (reduced stimulus; Figure $3 \mathrm{C}$ ). This manipulation did not augment the steady-state potential (ratio: $0.60 \pm 0.11$; Figure 3D). Taken together, these data suggest that presynaptic inhibition induced by DA is responsible for the larger steady-state field potential observed during HFS.

To directly examine the synaptic efficacy of TA-pyramidal neuron synapses, we made whole-cell voltage-clamp recordings from CA1 pyramidal neurons and measured current influx evoked by the TA pathway stimulation in control or DA-treated slices (Figure 4A). A comparison of the average TA-elicited EPSC waveforms from control versus DA-treated slices indicated no apparent differences in the EPSC waveform shape or kinetics (Figure 4A, left). Input resistance also did not differ between the groups. However, during HFS, current influx was significantly larger in the presence of DA (Figure 4A, middle and right), suggesting that synaptic efficacy of TA-pyramidal neuron synapses was enhanced by DA. To confirm that the above differences were caused by a modulation of inhibitory transmission, we made recordings under $\mathrm{GABA}_{\mathrm{A} \text { and } \mathrm{B}}$ receptor blockade to isolate excitatory inputs. We found that GABA receptor antagonists completely prevented the facilitation of the steady-state current by DA (Figure 4B), indicating that the observed difference (Figure 4A) was caused by inhibitory modulation. The above results reinforce the idea that DA-induced disinhibition enhances synaptic efficacy during HFS.

\section{DA imposes a high-pass filter on TA-CA1 synaptic transmission}

The oscillatory patterns of neural networks in the mammalian brain cover a wide range of frequencies approximately from 0.05 to $500 \mathrm{~Hz}$ (Buzsaki and Draguhn, 2004). We reasoned that the temporal features of disinhibition may interact with oscillatory dynamics of neural networks to produce frequency-dependent modulatory effects of DA. To delineate the frequency profile of DA-induced modulation, we stimulated the TA pathway with 100 pulses at frequencies ranging from 5 to $100 \mathrm{~Hz}$ (Figures 5A and 6A). Under control conditions, steady-state potentials became progressively smaller at stimulation frequencies greater than $10 \mathrm{~Hz}$ (Figure 6A). On the other hand, under the influence of DA, steady-state potentials were enhanced at all frequencies higher than $10 \mathrm{~Hz}$ (Figure 6A). Thus, DA exerts a stimulation frequency-dependent modulation of synaptic strength. In another set of analyses, we took into account the DA-induced inhibition of basal transmission by normalizing all potentials to the baseline fEPSP before DA application (Figures 5B and $6 B$ ). Under the influence of $D A$, steady-state potentials were smaller than control during low-frequency stimulation because of the excitatory depression, however, during HFS, the disinhibition overcame the depression and steady-state potentials were larger than control (Figure 6B). A similar of results was obtained in a set of experiments conducted at near physiological temperature $\left(32-34^{\circ} \mathrm{C}\right.$ ) (Figures 6C and 6D). These data indicate that the DA-induced disinhibition together 


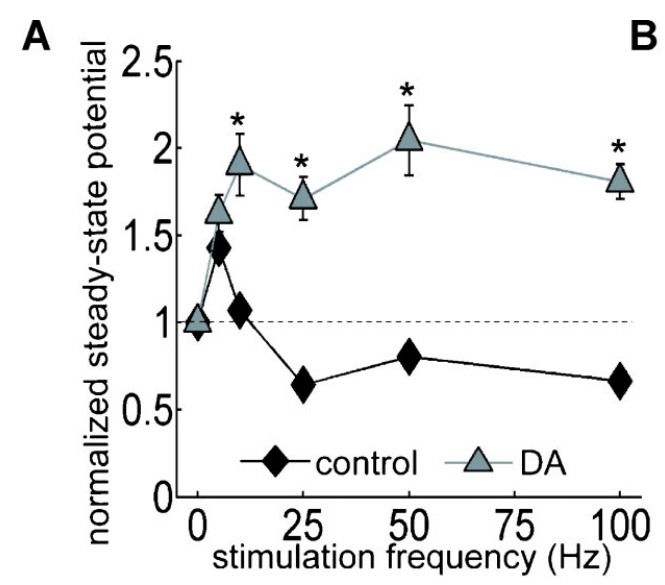

C

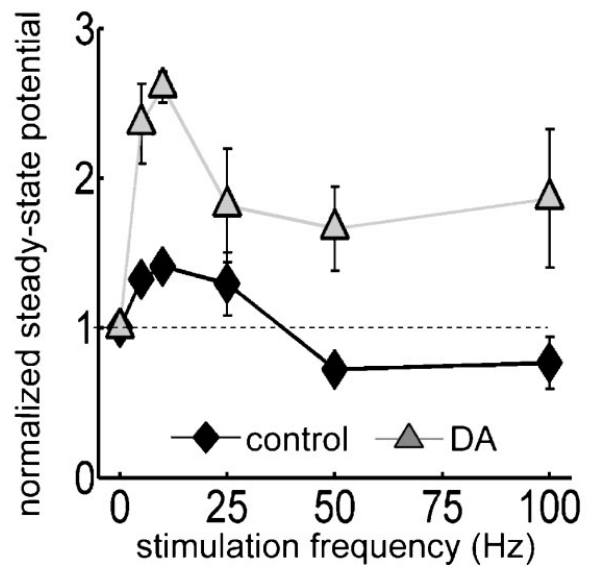

B

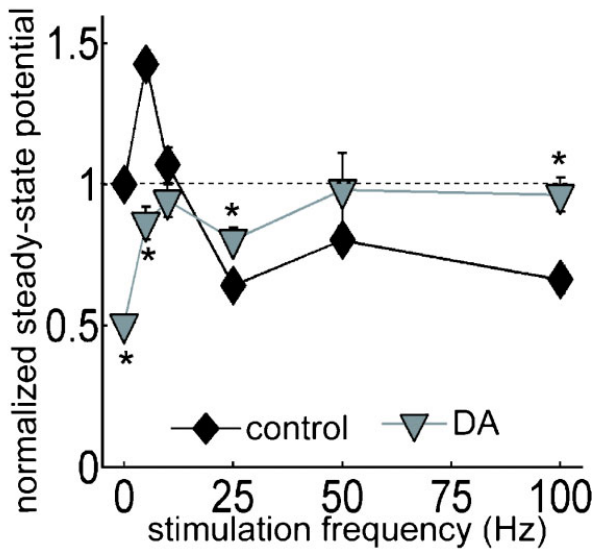

D

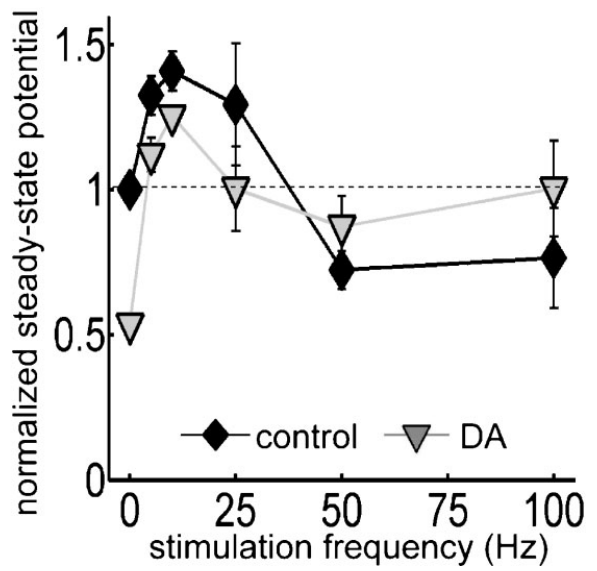

Figure 6. Analysis of DA-induced filtering at TA-CA1 synapses at room and near physiological temperatures. (A) Using same data as in Figure 5, steady-state potentials were measured. In the presence of DA, the steady-state potential became larger during HFS. $p<0.05$ relative to control (from left to right; control: $n=5,5,7,6$, and 5, DA: $n=5,5,5,5$, and 5). (B) As in Figure 5B, data were normalized to baseline fEPSP amplitude prior to DA application. Thus, this figure shows the total effect of DA, including on the depression of basal synaptic transmission. Under DA application, although the steady-state potential was smaller during low-frequency stimulation, it overcame the depression and became larger than control during HFS. $p<0.05$ relative to control. Note that control error bars are smaller than the symbol size. (C) Similar experiments as $\boldsymbol{A}$ conducted at higher temperature (32-34 $\mathrm{C})$. (D) Similar analysis as $\boldsymbol{B}$, using data acquired at higher temperature.

with its excitatory depression exhibits an alternate gating of synaptic strength in a frequency-dependent manner-increasing the impact of high-frequency inputs while decreasing the impact of low-frequency signals. Thus, DA acts as a high-pass filter on TA-CA1 pyramidal neuron signaling.

\section{DA bi-directionally modulates synaptic plasticity}

To assess the functional impact of DA-induced filtering, we next examined the DA's effect on synaptic plasticity, the sign and strength of which is known to be dependent on stimulation frequency. To test this idea, LTP was induced at TA-CA1 synapses with or without DA (Figure 7A). LTP, induced by HFS (5 trains of $100 \mathrm{~Hz} 100$ pulses), was significantly enhanced by DA (control: $126.4 \pm 7.2$, DA: $172.6 \pm 14.3,50-60$ minute after LTP induction), which was blocked by DA receptor antagonists (Figures 7D and 7E). On the other hand, LTD, which is induced by low-frequency stimulation, was attenuated by DA (Figure 7B; control: $81.7 \pm 3.5 \%$, DA: $94.8 \pm 2.3 \%, 30-35$ minute after LTD induction). Thus, the frequency-dependent signal filtering by DA has a profound functional impact on the magnitude of synaptic plasticity. We next examined whether modulation of inhibitory transmission also underlies this phenomena. Under GABA receptor blockade, LTP at TA-CA1 synapses was enhanced, and DA's effect on LTP enhancement was occluded (Figure 7C; Bic + CGP: $171.5 \pm 18.6 \%$, Bic + CGP + DA: $152.1 \pm 9.8 \%, 50-60$ minute after LTP induction), suggesting an obligatory contribution from the inhibitory network.

How quickly can the network adapt to DA signals? Because dopaminergic neurons show both tonic and burst-like activity patterns in vivo (Floresco et al., 2003; Grace, 1991), we examined the sensitivity of this modulation to very brief ( 10 second $+1-2$ minute washout) temporally controlled applications of DA (Figures $8 \mathrm{~A}$ and $8 \mathrm{~B}$ ). When DA was applied 10 second before LTP induction, LTP was significantly enhanced when compared with vehicle-applied control (Figure 8C; vehicle: $107.0 \pm 2.7 \%$, DA: $136.5 \pm 8.7 \%, 50-60$ minute after LTP induction). The application of DA for 10 second 3 minute before LTP or 10 second after induction, however, did not enhance of TA-CA1 LTP (Figure 8D; 3 minute before: $111.0 \pm 4.0 \%, 10$ second after: $110.7 \pm 5.6 \%)$. These data indicate that extremely brief DA application that is coincident with LTP induction is capable of modulating the TA-CA1 plasticity network. 

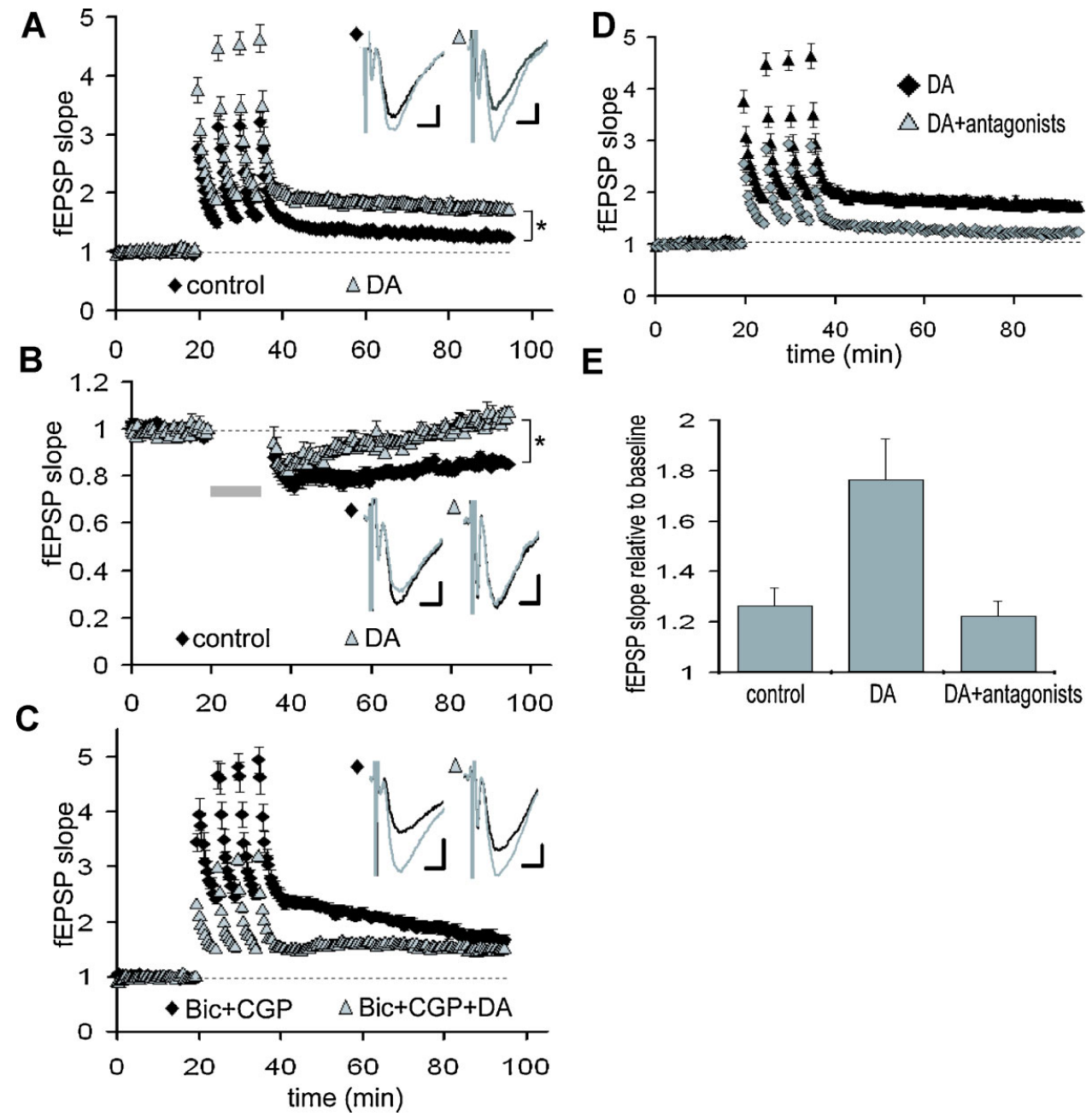

Figure 7. DA-induced modulation of synaptic plasticity at TA-CA1 synapses. (A) Enhancement of TA-CA1 LTP by DA. DA was present for the duration of the experiment. Baselines are normalized to 1.0 in order to examine LTP independent of the depression of basal synaptic transmission by DA. For all traces, the baseline (preplasticity) trace is black, the post-plasticity trace is gray. $p<0.01$ (control: $n=7, D A: n=8$ ). Scale bar $=0.1 \mathrm{mV}, 5 \mathrm{~ms}$. (B) Attenuation of TA-CA1 LTD by DA. Gray bar: LTD induction. $p<0.01$ (control: $n=7, D A: n=6$ ). Scale bar $=0.1 \mathrm{mV}, 5 \mathrm{~ms}$. (C) Occlusion of DA-induced TA-CA1 LTP enhancement under GABA $A_{A \text { and } B}$ receptor blockade. Under GABA receptor blockade, DA did not significantly enhance LTP ( $n=5$ for each group). Scale bar $=0.1 \mathrm{mV}, 5 \mathrm{~ms}$. (D) Blockade of DA-induced enhancement of TA-LTP by DA receptor antagonists, SKF $83566(1 \mu M)$ and Sulpiride (10 $\mu M)(D A: n=8, D A$ antagonists: $n=5)$. (E) Analysis of LTP at TA-CA1 synapses (50-60minute after LTP induction). DA receptor antagonists completely blocked DA-induced enhancement of TA-LTP (control: $n=7, D A: n=8, D A$ antagonists: $n=5$ ).

\section{DA-induced disinhibition influences synaptic plasticity at SC-CA1 synapses}

Although DA-induced excitatory depression is selective to TA-CA1 synapses, the resulting disinhibition may influence another excitatory input to area CA1, the SC pathway. Previous work has shown that TA activity can interfere with LTP induction at SC-CA1 synapses, primarily due to inhibition evoked by the TA pathway stimulation (Levy et al., 1998; Remondes and Schuman, 2002). We reasoned that DA-induced disinhibition may attenuate this LTP interference. We first confirmed that DA had no significant effect on the magnitude of LTP at SC-CA1 synapses up to 1 hour after LTP induction, when the SC was stimulated alone either by TBS (Figure 9C; control: $142.4 \pm 5.7 \%$, DA: $139.7 \pm 5.3 \%, 50-60$ minute after LTP induction) or by HFS (Figure 9D; control: $179.6 \pm 15.5 \%$, DA: $179.5 \pm 13.0 \%$ ). As previously shown, joint TBS applied to both the SC and TA pathways significantly attenuated LTP at SC-CA1 synapses (Figure 9E; $112.3 \pm 3.0 \%$ ). To assess the DA-induced disinhibitory effect on LTP inter- ference, the stimulation amplitude was increased after DA application to compensate for the DA-induced excitatory depression (Figure 9B). Under this condition, DA significantly attenuated the LTP interference of SC-CA1 synapses elicited by concurrent TA activity (Figure 9E; $128.0 \pm 1.5 \%$ ). These results indicate that DA-induced disinhibition has a large impact on plasticity induction not only at TA-CA1 synapses, but also at SC-CA1 synapses.

\section{DISCUSSION}

\section{Frequency-dependent signal filtering by DA}

Our results indicate that DA imposes a high-pass filter on signal propagation at TA-CA1 synapses and dynamically modulates information flow and synaptic plasticity in area CA1. Although previous studies have shown synaptic modulation induced by specific DA receptor subtypes in the hippocampus (Chen etal., 1996; Huang and Kandel, 1995; 
A

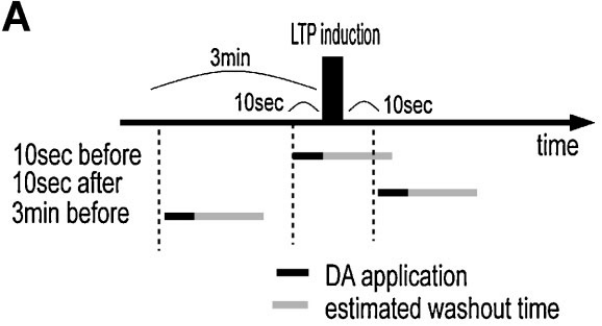

C

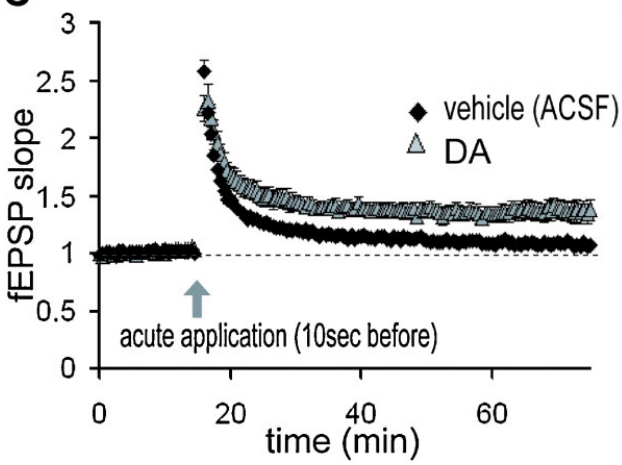

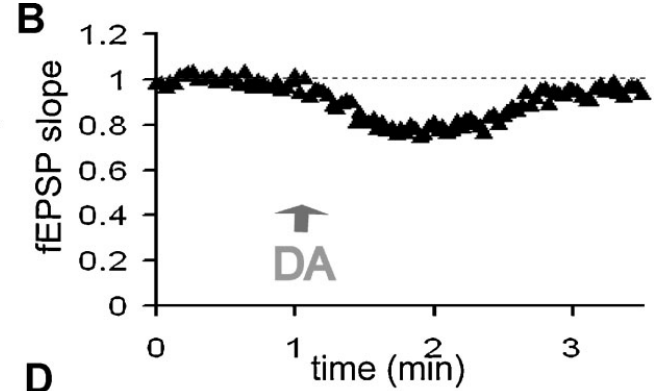

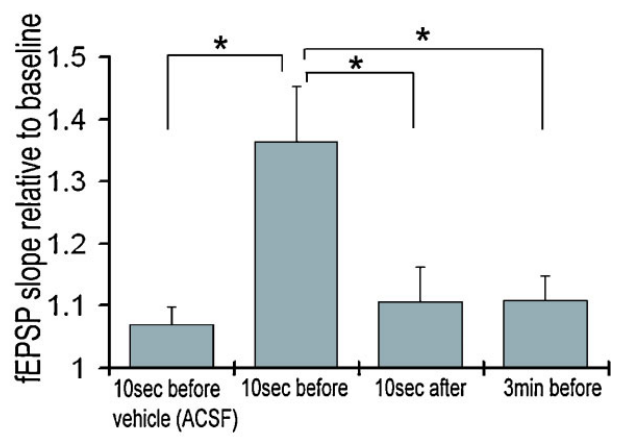

Figure 8. Temporally selectivity of LTP enhancement by DA. (A) Acute application of DA. DA was applied for just 10 second either 10 second before, 10 second after, or 3 minute before LTP induction. LTP induction protocol was $100 \mathrm{~Hz} 100$ pulse stimulation, repeated 2 times at an interval of 30 second. DA was directly applied in the recording chamber for 10 second and estimated washout time was about 1-2 minute. (B) fEPSP slopes during acute application of DA. TA pathway was stimulated at $0.5 \mathrm{~Hz}$. DA was directly applied into the recording chamber at 1 minute (indicated by arrow) and its effects on synaptic transmission were reversible. (C) DA application at 10 second before LTP induction significantly enhanced LTP at TACA1 synapses (vehicle: $n=7, D A: n=8$ ). (D) DA application 3 minute before or 10 second after LTP induction did not enhance LTP (bottom, $n=6$ for each).

Otmakhova and Lisman, 1996, 1998), it was not clear how DA itself, as a neurotransmitter, modulates the activity of the integrated hippocampal network. Because of complex cooperative and uncooperative interactions among DA receptor subtypes (Lee et al., 2004; Missale et al., 1998; Plaznik etal., 1989; Rashid etal., 2007; Schmidt and Pierce, 2006; Taussig etal., 1993; Tucek etal., 2002; Wu and Hablitz, 2005), we decided to examine, primarily, the combined effects resulting from application of DA itself. Here we demonstrate that DA has a profound influence on signal integration of the two pathways in hippocampal area CA1, which may represent an information selection process for learning.

Because both synaptic transmission and action potential generation depend nonlinearly on the input patterns, it is important to understand synaptic function over a wide range of activity frequencies. Indeed, the importance of a frequency-dependent analysis of synaptic transmission was previously suggested (Markram etal., 1998). The frequencydependent filter at TA-CA1 synapses we describe here, differentially modulates (i.e., depresses or enhances) signals depending on input frequency, thus transferring qualitatively different information through the synaptic network. This frequency-dependency emerges due to differential responses to synaptic inputs that result from monosynaptic and disynaptic transmission (Figures 3B and 10). One advantage of this type of alternate gating is the potential for enabling dynamic coupling among different brain areas, because of actively generated oscillatory activities in the brain (Buzsaki and Draguhn, 2004; Siapas et al., 2005). Dynamic modulation of information flow via oscillatory coupling is suggested to have an important role in learning (for review, Axmacher et al., 2006).

\section{Functional impact of high-pass signal filtering on in vivo oscillatory activities}

Mutual interactions between the dopaminergic system and the hippocampus have been previously suggested (Lisman and Grace, 2005; Lisman and Otmakhova, 2001). Both the hippocampus and the dopaminergic system show differential activation depending on the familiarity of the stimuli (Fyhn et al., 2002; Horvitz, 2000; Kumaran and Maguire, 2006; Rutishauser et al., 2006; Schultz, 1998; Vinogradova, 2001) and influence learning (Adcock et al., 2006; Buhusi et al., 1998; Katz et al., 2002; Meltzer and Constable, 2005; Wittmann et al., 2005). In contrast with short-latency responses $(<100 \mathrm{~ms})$ of dopaminergic neurons to the stimuli (Comoli etal., 2003; Dommett et al., 2005; Schultz, 1998), differential activation of the hippocampus appears approximately from 200 to $400 \mathrm{~ms}$ after the stimuli (Brankack et al., 1996; Grunwald etal., 1998; Knight, 1996), suggesting DA may serve as a gate to modulate information flow into the hippocampus (Black et al., 2000; Jurkowlaniec et al., 2003; Orzel-Gryglewska et al., 2006).

Our demonstration of frequency-dependent signal modulation by the neurotransmitter DA has important ramifications for network function in vivo, especially when one considers the behavior-dependent oscillatory activities emerging from different brain structures (Buzsaki, 1996, 2002; Steriade, 2001). For example, during exploratory behavior, the firing patterns of neurons in layer II/III of the entorhinal cortex which send projections to the hippocampus, are characterized by theta $(4-12 \mathrm{~Hz})$ and gamma frequency volleys $(40-100 \mathrm{~Hz}$ ) (Chrobak et al., 2000). Our data predict that precisely these frequencies will be differentially influenced by DA-induced high-pass filtering. Because signals from the entorhinal cortex 

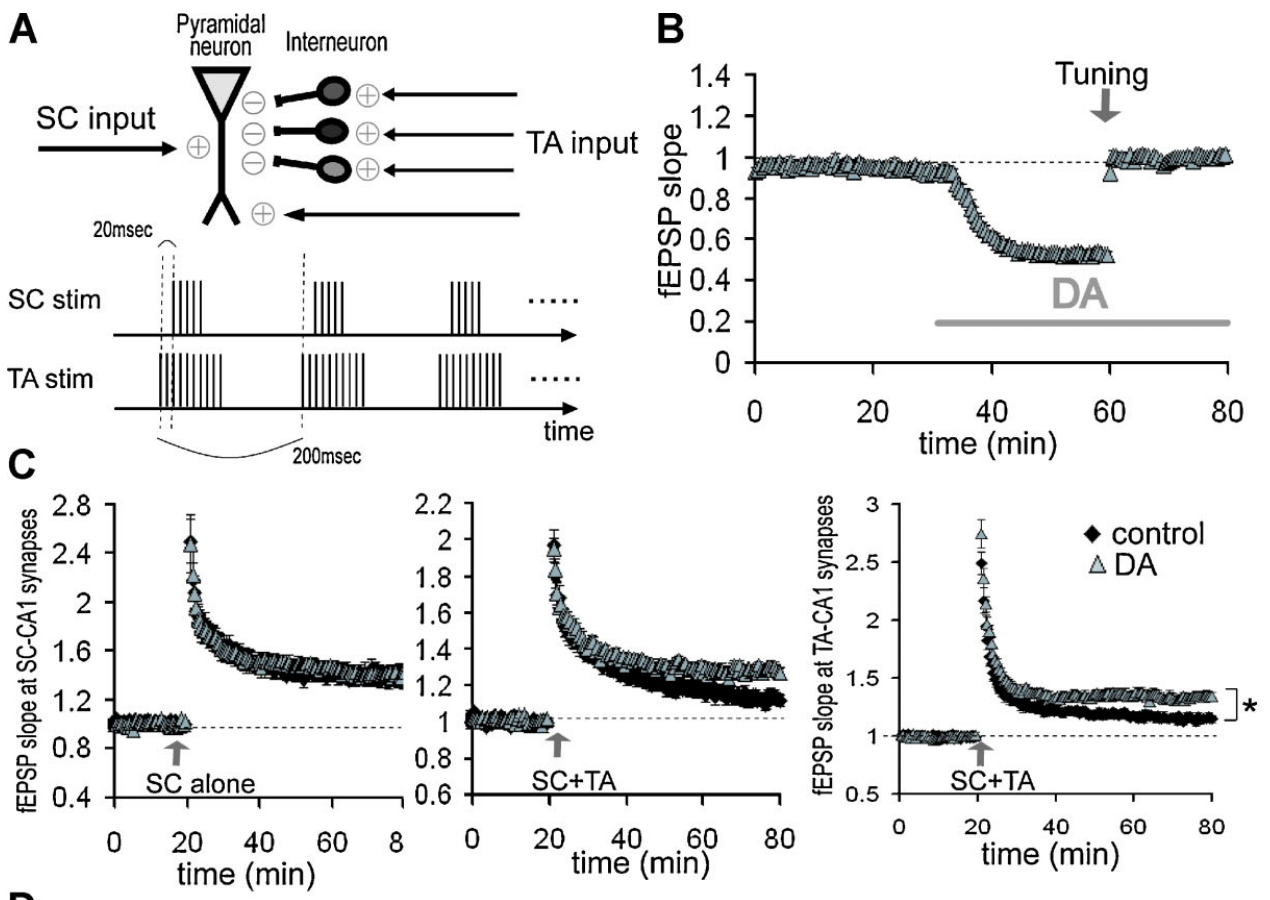

D
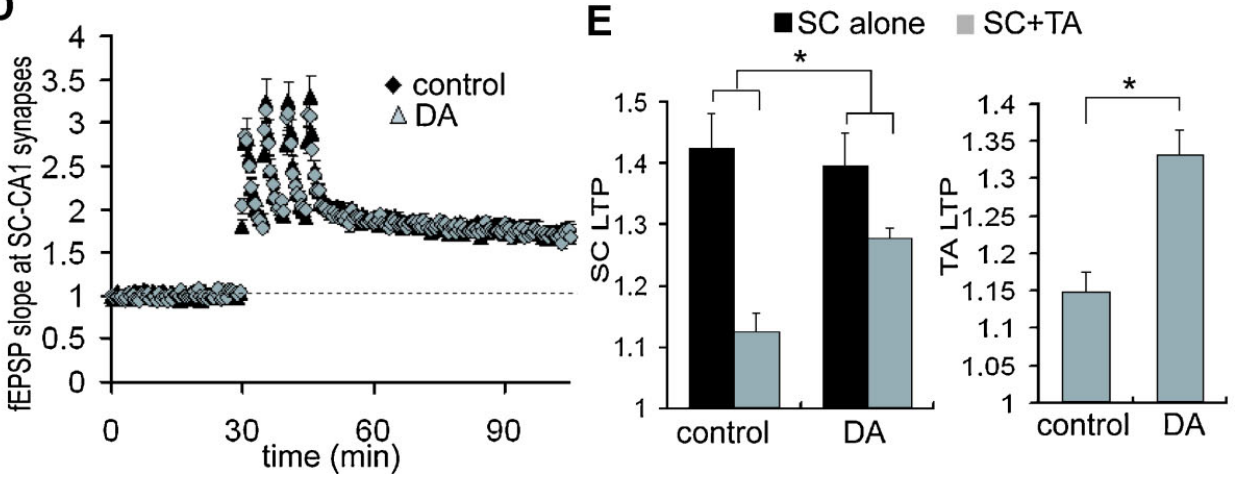

Figure 9. Reduction of LTP interference at SC-CA1 synapses by DA-induced disinhibition at TA-CA1 synapses. (A) Scheme of LTP interference protocol. Bursts (10) of either 5 (SC) or 10 (TA) at $100 \mathrm{~Hz}$ with $200 \mathrm{~ms}$ interburst interval, was repeated twice at 30 second interval. TA-TBS precedes SC-TBS by $20 \mathrm{~ms}$. (B) Compensation of DA-induced excitatory depression by increasing the stimulation current. After DA-induced depression was stabilized, the stimulation current was increased to return the fEPSP to its baseline value (tuning, indicated by arrow). (C) Influence of DA on the LTP interference of SCCA1 synapses elicited by the TA pathway stimulation. TBS was applied to either the SC pathway alone (left; control: $n=8, D A: n=6)$ or both the SC and the TA pathways concurrently (middle; $n=9$ for each group). Right figure shows the enhancement of LTP at TA-CA1 synapses by DA after concurrent TBS application ( $n=9$ for each group). (D) No significant influence of DA on SC-LTP induced by HFS. LTP induction protocol was $100 \mathrm{~Hz}$ (100 pulse) stimulation, repeated 4 times at 5 minute intervals ( $n=6$ for each). (E) Analysis of DA's effects on the magnitude of LTP after TBS application, either at SC-CA1 synapses (left) or at TA-CA1 synapses. DA significantly attenuated the LTP interference at SC-CA1 synapses $(p<0.05)$, and enhanced LTP at TA-CA1 synapses $(p<0.01)$

(the TA pathway) are thought to relay sensory-bound information (Quirk et al., 1992; Witter and Moser, 2006), in contrast with the associatively processed information from area CA3 (the SC pathway) (Nakazawa et al., 2002; Treves and Rolls, 1994), the DA-induced frequency-selective enhancement of TA-CA1 signals may modulate the integration of environmental information with previously associated information as it leaves the hippocampus through area CA1. Supporting this idea, a theoretical study suggests that input frequency at TA-CA1 synapses will influence the decoding of information carried via the SC pathway (Yoshida et al., 2002). Furthermore, we demonstrate that DA-induced disinhibition attenuates the TA-evoked interference of plasticity induction at SC-CA1 synapses, possibly boosting information integration in area CA1. Taken together, these data indicate that DA can transform the hippocampal network to an "encoding mode" by reducing the inhibition on CA1 pyramidal neurons and modulating the information flow from the entorhinal cortex, thus facilitating the learning of behaviorally relevant events.

\section{CONFLICT OF INTEREST STATEMENT}

This research was conducted in the absence of any commercial or financial relationships that could be construed as a potential conflict of interest.

\section{ACKNOWLEDGEMENTS}

We thank members of the Schuman laboratory for discussions. H. T. I. is supported by the Nakajima foundation. E. M. S. is an investigator of the Howard Hughes Medical Institute. 
A

Simulated Response to $100 \mathrm{~Hz}$ stimulation
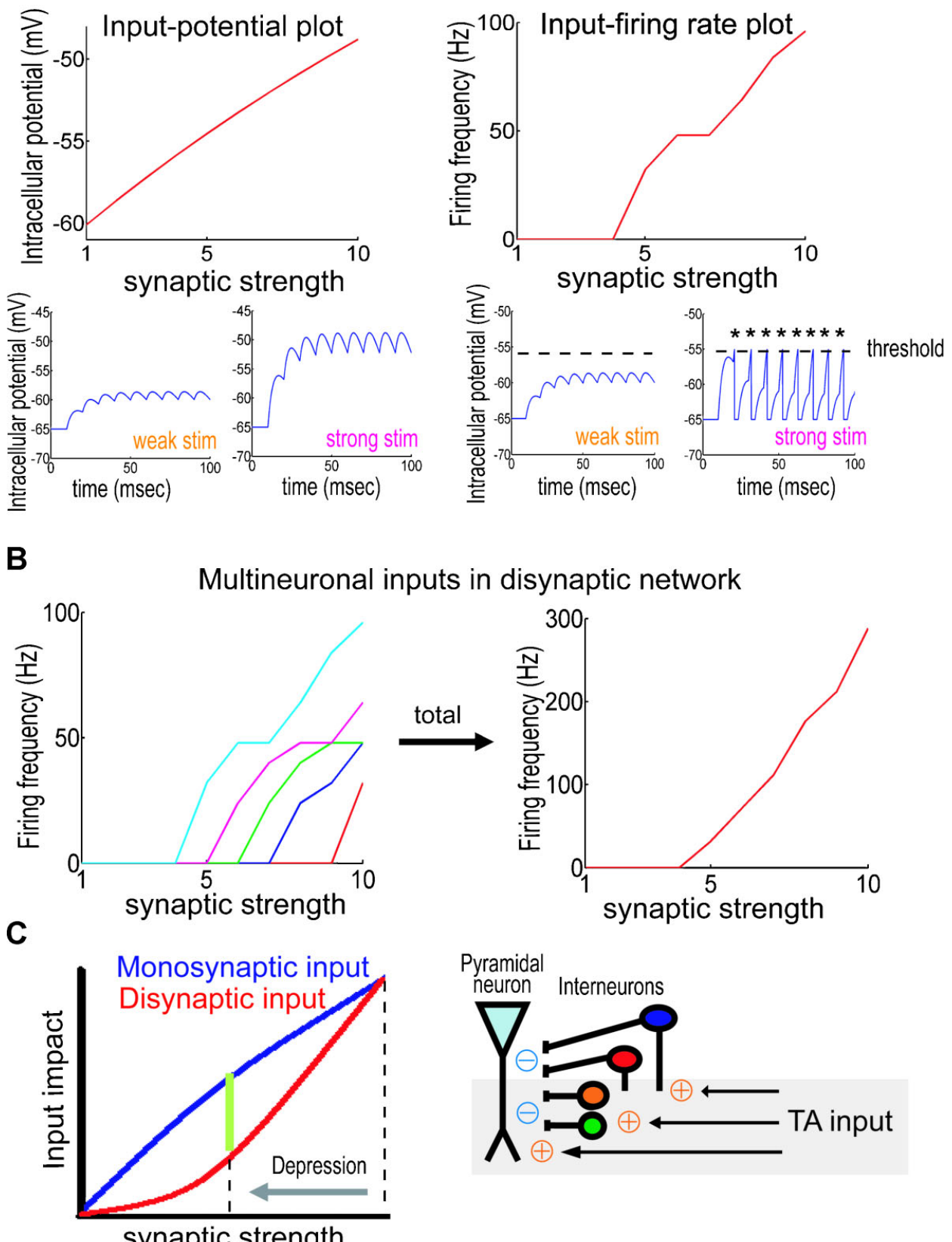

Figure 10. Modulation of excitatory/inhibitory balance by DA-induced excitatory depression. (A) Simulation based on an integrate-and-fire model. Excitatory stimuli at $100 \mathrm{~Hz}$ are delivered using EPSPS of different initial strengths. Each plot shows either maximal membrane potential (left) or firing frequency (right) to a given initial input strength. During monosynaptic transmission, maximum membrane potentials depend approximately linearly on input strength (left). Disynaptic transmission, on the other hand, requires action potential generation for the signal propagation, which imposes a nonlinear dependency on input strength (right). (B) Total signal output from multiple neurons. Each neuron has a different threshold-level as plotted in different colors (left). Summated output depends nonlinearly on input synaptic strength (right). Similarly, at TA-CA1 synapses, a pyramidal neuron receives disynaptic inhibition from multiple number of interneurons, thus inhibition evoked by the TA pathway should nonlinearly depend on the input synaptic strength. (C) Differential influence of synaptic depression between monosynaptic and disynaptic transmission. After depression of TA-CA1 synaptic transmission, the reduction of disynaptic inhibition is larger than that of monosynaptic excitation, explaining why the synaptic efficacy is enhanced during HFS, as observed as high-pass filtering, under the DA-induced excitatory depression. Other interneuron classes not studied here may be similarly affected by DA.

\section{REFERENCES}

Adcock, R. A., Thangavel, A., Whitfield-Gabrieli, S., Knutson, B., and Gabrieli, J. D. (2006). Reward-motivated learning: mesolimbic activation precedes memory formation. Neuron 50, 507-517.

Axmacher, N., Mormann, F., Fernandez, G., Elger, C. E., and Fell, J. (2006). Memory formation by neuronal synchronization. Brain Res. Rev. 52, 170-182.
Black, K. J., Hershey, T., Gado, M. H., and Perlmutter, J. S. (2000). Dopamine D(1) agonist activates temporal lobe structures in primates. J. Neurophysiol. 84, 549-557.

Brankack, J., Seidenbecher, T., and Muller-Gartner, H. W. (1996). Task-relevant late positive component in rats: is it related to hippocampal theta rhythm? Hippocampus $6,475-482$

Brun, V. H., Otnass, M. K., Molden, S., Steffenach, H. A., Witter, M. P., Moser, M. B., and Moser, E. I. (2002). Place cells and place 
recognition maintained by direct entorhinal-hippocampal circuitry. Science 296 , 2243-2246.

Buhusi, C. V., Gray, J. A., and Schmajuk, N. A. (1998). Perplexing effects of hippocampal lesions on latent inhibition: a neural network solution. Behav. Neurosci. 112, 316-351.

Buzsaki, G. (1996). The hippocampo-neocortical dialogue. Cereb. Cortex 6, 81-92.

Buzsaki, G. (2002). Theta oscillations in the hippocampus. Neuron 33, 325-340.

Buzsaki, G., and Draguhn, A. (2004). Neuronal oscillations in cortical networks. Science 304, 1926-1929.

Cajal, S. R. Y. (1911). Histologie du Systeme nerveux de l'hommes et des vertebres, Vol II (Paris, Maloine).

Chen, Z., Ito, K., Fujii, S., Miura, M., Furuse, H., Sasaki, H., Kaneko, K., Kato, H., and Miyakawa, H. (1996). Roles of dopamine receptors in long-term depression: enhancement via D1 receptors and inhibition via D2 receptors. Recept. Channels 4 , $1-8$.

Chrobak, J. J., Lorincz, A., and Buzsaki, G. (2000). Physiological patterns in the hippocampo-entorhinal cortex system. Hippocampus 10, 457-465.

Comoli, E., Coizet, V., Boyes, J., Bolam, J. P., Canteras, N. S., Quirk, R. H., Overton, P. G., and Redgrave, P. (2003). A direct projection from superior colliculus to substantia nigra for detecting salient visual events. Nat. Neurosci. 6, 974-980.

Desmond, N. L., Scott, C. A., Jane, J. A., Jr., and Levy, W. B. (1994). Ultrastructural identification of entorhinal cortical synapses in CA1 stratum lacunosum-moleculare of the rat. Hippocampus 4, 594-600.

Dobrunz, L. E., and Stevens, C. F. (1997). Heterogeneity of release probability, facilitation, and depletion at central synapses. Neuron 18, 995-1008.

Dommett, E., Coizet, V., Blaha, C. D., Martindale, J., Lefebvre, V., Walton, N., Mayhew, J. E., Overton, P. G., and Redgrave, P. (2005). How visual stimuli activate dopaminergic neurons at short latency. Science 307, 1476-1479.

Dvorak-Carbone, H., and Schuman, E. M. (1999a). Long-term depression of temporoammonic-CA1 hippocampal synaptic transmission. J. Neurophysiol. 81 1036-1044.

Dvorak-Carbone, H., and Schuman, E. M. (1999b). Patterned activity in stratum lacunosum moleculare inhibits CA1 pyramidal neuron firing. J. Neurophysiol. 82 , 3213-3222.

Empson, R. M., and Heinemann, U. (1995). The perforant path projection to hippocampa area CA1 in the rat hippocampal-entorhinal cortex combined slice. J. Physiol. 484 (Pt 3), 707-720.

Floresco, S. B., West, A. R., Ash, B., Moore, H., and Grace, A. A. (2003). Afferent modulation of dopamine neuron firing differentially regulates tonic and phasic dopamine transmission. Nat. Neurosci. 6, 968-973.

Freund, T. F., and Buzsaki, G. (1996). Interneurons of the hippocampus. Hippocampus 6, 347-470.

Fyhn, M., Molden, S., Hollup, S., Moser, M. B., and Moser, E. (2002). Hippocampal neurons responding to first-time dislocation of a target object. Neuron 35, 555-566.

Gasbarri, A., Packard, M. G., Campana, E., and Pacitti, C. (1994a). Anterograde and retrograde tracing of projections from the ventral tegmental area to the hippocampa formation in the rat. Brain Res. Bull. 33, 445-452.

Gasbarri, A., Packard, M. G., Sulli, A., Pacitti, C., Innocenzi, R., and Perciavalle, V. (1996a) The projections of the retrorubral field $A 8$ to the hippocampal formation in the rat Exp. Brain Res. 112, 244-252.

Gasbarri, A., Sulli, A., Innocenzi, R., Pacitti, C., and Brioni, J. D. (1996b). Spatial memory impairment induced by lesion of the mesohippocampal dopaminergic system in the rat. Neuroscience 74, 1037-1044.

Gasbarri, A., Sulli, A., and Packard, M. G. (1997). The dopaminergic mesencephalic projections to the hippocampal formation in the rat. Prog Neuropsychopharmacol. Biol. Psychiatry 21, 1-22.

Gasbarri, A., Verney, C., Innocenzi, R., Campana, E., and Pacitti, C. (1994b). Mesolimbic dopaminergic neurons innervating the hippocampal formation in the rat: combined retrograde tracing and immunohistochemical study. Brain Res. 668, $71-79$.

Grace, A. A. (1991). Phasic versus tonic dopamine release and the modulation of dopamine system responsivity: a hypothesis for the etiology of schizophrenia. Neuroscience 41, 1-24.

Grunwald, T., Lehnertz, K., Heinze, H. J., Helmstaedter, C., and Elger, C. E. (1998). Verbal novelty detection within the human hippocampus proper. Proc. Natl. Acad. Sci. USA 95, 3193-3197.

Guzowski, J. F., Knierim, J. J., and Moser, E. I. (2004). Ensemble dynamics of hippocampal regions CA3 and CA1. Neuron 44, 581-584.

Hasselmo, M. E., and Schnell, E. (1994). Laminar selectivity of the cholinergic suppression of synaptic transmission in rat hippocampal region CA1: computational modeling and brain slice physiology. J. Neurosci. 14, 3898-3914.

Hasselmo, M. E., Wyble, B. P., and Wallenstein, G. V. (1996). Encoding and retrieval of episodic memories: role of cholinergic and GABAergic modulation in the hippocampus. Hippocampus 6, 693-708.

Hopf, F. W., Cascini, M. G., Gordon, A. S., Diamond, I., and Bonci, A. (2003). Cooperative activation of dopamine D1 and D2 receptors increases spike firing of nucleus accumbens neurons via G-protein betagamma subunits. J. Neurosci. 23 , 5079-5087.

Horvitz, J. C. (2000). Mesolimbocortical and nigrostriatal dopamine responses to salient non-reward events. Neuroscience 96, 651-656.

Huang, Y. Y., and Kandel, E. R. (1995). D1/D5 receptor agonists induce a protein synthesisdependent late potentiation in the CA1 region of the hippocampus. Proc. Natl. Acad. Sci. USA 92, 2446-2450.
Ihalainen, J. A., Riekkinen, P., Jr., and Feenstra, M. G. (1999). Comparison of dopamine and noradrenaline release in mouse prefrontal cortex, striatum and hippocampus using microdialysis. Neurosci. Lett. 277, 71-74.

Ikemoto, S., Glazier, B. S., Murphy, J. M., and McBride, W. J. (1997). Role of dopamine D1 and D2 receptors in the nucleus accumbens in mediating reward. J. Neurosci. 17, 8580-8587.

Jurkowlaniec, E., Tokarski, J., and Trojniar, W. (2003). Effect of unilateral ibotenate lesions of the ventral tegmental area on cortical and hippocampal EEG in freely behaving rats. Acta Neurobiol. Exp. (Wars) 63, 369-375.

Katz, B., and Miledi, R. (1968). The role of calcium in neuromuscular facilitation. J. Physiol. 195, 481-492.

Katz, D. B., Rogers, R. F., and Steinmetz, J. E. (2002). Novel factors contributing to the expression of latent inhibition. Behav. Neurosci. 116, 824-836.

Knierim, J. J., Lee, I., and Hargreaves, E. L. (2006). Hippocampal place cells: parallel input streams, subregional processing, and implications for episodic memory. Hippocampus 16, 755-764.

Knight, R. (1996). Contribution of human hippocampal region to novelty detection. Nature $383,256-259$.

Kumaran, D., and Maguire, E. A. (2006). An unexpected sequence of events: mismatch detection in the human hippocampus. PLoS Biol. 4, e424.

Lacaille, J. C., and Schwartzkroin, P. A. (1988). Stratum lacunosum-moleculare interneurons of hippocampal CA1 region. II. Intrasomatic and intradendritic recordings of local circuit synaptic interactions. J. Neurosci. 8, 1411-1424.

Lee, I., Yoganarasimha, D., Rao, G., and Knierim, J. J. (2004a). Comparison of population coherence of place cells in hippocampal subfields CA1 and CA3. Nature 430 456-459.

Lee, S. P., So, C. H., Rashid, A. J., Varghese, G., Cheng, R., Lanca, A. J., O'Dowd, B. F. and George, S. R. (2004b). Dopamine D1 and D2 receptor Co-activation generates a novel phospholipase C-mediated calcium signal. J. Biol. Chem. 279, 35671-35678.

Leutgeb, S., Leutgeb, J. K., Treves, A., Moser, M. B., and Moser, E. I. (2004). Distinct ensemble codes in hippocampal areas CA3 and CA1. Science 305, 1295-1298.

Levy, W. B., Desmond, N. L., and Zhang, D. X. (1998). Perforant path activation modulates the induction of long-term potentiation of the schaffer collateral-hippocampal CA1 response: theoretical and experimental analyses. Learn Mem. 4, 510-518.

Lisman, J. E., and Grace, A. A. (2005). The hippocampal-VTA loop: controlling the entry of information into long-term memory. Neuron 46, 703-713.

Lisman, J. E., and Otmakhova, N. A. (2001). Storage, recall, and novelty detection of sequences by the hippocampus: elaborating on the SOCRATIC model to account for normal and aberrant effects of dopamine. Hippocampus 11, 551-568.

Markram, H., Gupta, A., Uziel, A., Wang, Y., and Tsodyks, M. (1998). Information processing with frequency-dependent synaptic connections. Neurobiol. Learn Mem. 70, 101-112.

McClure, S. M., Daw, N. D., and Montague, P. R. (2003). A computational substrate for incentive salience. Trends Neurosci. 26, 423-428.

Meltzer, J. A., and Constable, R. T. (2005). Activation of human hippocampal formation reflects success in both encoding and cued recall of paired associates. Neuroimage 24, 384-397.

Missale, C., Nash, S. R., Robinson, S. W., Jaber, M., and Caron, M. G. (1998). Dopamine receptors: from structure to function. Physiol. Rev. 78, 189-225.

Montague, P. R., Hyman, S. E., and Cohen, J. D. (2004). Computational roles for dopamine in behavioural control. Nature 431, 760-767.

Nakazawa, K., Quirk, M. C., Chitwood, R. A., Watanabe, M., Yeckel, M. F., Sun, L. D., Kato A., Carr, C. A., Johnston, D., Wilson, M. A., and Tonegawa, S. (2002). Requirement for hippocampal CA3 NMDA receptors in associative memory recall. Science 297, 211-218

Orzel-Gryglewska, J., Jurkowlaniec, E., and Trojniar, W. (2006). Microinjection of procaine and electrolytic lesion in the ventral tegmental area suppresses hippocampal theta rhythm in urethane-anesthetized rats. Brain Res. Bull. 68, 295-309.

Otmakhova, N. A., Lewey, J., Asrican, B., and Lisman, J. E. (2005). Inhibition of perforant path input to the CA1 region by serotonin and noradrenaline. J. Neurophysiol. 94 1413-1422.

Otmakhova, N. A., and Lisman, J. E. (1996). D1/D5 dopamine receptor activation increases the magnitude of early long-term potentiation at CA1 hippocampa synapses. J. Neurosci. 16, 7478-7486.

Otmakhova, N. A., and Lisman, J. E. (1998). D1/D5 dopamine receptors inhibit depotentiation at CA1 synapses via CAMP-dependent mechanism. J. Neurosci. 18 , 1270-1279.

Otmakhova, N. A., and Lisman, J. E. (1999). Dopamine selectively inhibits the direct cortical pathway to the CA1 hippocampal region. J. Neurosci. 19, 1437-1445.

Plaznik, A., Stefanski, R., and Kostowski, W. (1989). Interaction between accumbens D1 and D2 receptors regulating rat locomotor activity. Psychopharmacology (Berl.) 99 $558-562$

Quirk, G. J., Muller, R. U., Kubie, J. L., and Ranck, J. B., Jr. (1992). The positional firing properties of medial entorhinal neurons: description and comparison with hippocampal place cells. J. Neurosci. 12, 1945-1963.

Rashid, A. J., So, C. H., Kong, M. M., Furtak, T., El-Ghundi, M., Cheng, R., O'Dowd, B. F., and George, S. R. (2007). D1-D2 dopamine receptor heterooligomers with unique pharmacology are coupled to rapid activation of $\mathrm{Gq} / 11$ in the striatum. Proc. Natl. Acad Sci. USA 104, 654-659.

Remondes, M., and Schuman, E. M. (2002). Direct cortical input modulates plasticity and spiking in CA1 pyramidal neurons. Nature 416, 736-740.

Remondes, M., and Schuman, E. M. (2004). Role for a cortical input to hippocampal area CA1 in the consolidation of a long-term memory. Nature 431, 699-703. 
Rutishauser, U., Mamelak, A. N., and Schuman, E. M. (2006). Single-trial learning of novel stimuli by individual neurons of the human hippocampus-amygdala complex. Neuron 49, 805-813.

Schmidt, H. D., and Pierce, R. C. (2006). Cooperative activation of D1-like and D2-like dopamine receptors in the nucleus accumbens shell is required for the reinstatement of cocaine-seeking behavior in the rat. Neuroscience 142 $451-461$.

Schultz, W. (1998). Predictive reward signal of dopamine neurons. J. Neurophysiol. 80 $1-27$

Schultz, W., and Dickinson, A. (2000). Neuronal coding of prediction errors. Annu. Rev Neurosci. 23, 473-500.

Scoville, W. B., and Milner, B. (1957). Loss of recent memory after bilateral hippocampal lesions. J. Neurochem. 20, 11-21.

Siapas, A. G., Lubenov, E. V., and Wilson, M. A. (2005). Prefrontal phase locking to hippocampal theta oscillations. Neuron 46, 141-151.

Squire, L. R., Stark, C. E., and Clark, R. E. (2004). The medial temporal lobe. Annu. Rev. Neurosci. 27, 279-306.

Steffenach, H. A., Sloviter, R. S., Moser, E. I., and Moser, M. B. (2002). Impaired retention of spatial memory after transection of longitudinally oriented axons of hippocampal CA3 pyramidal cells. Proc. Natl. Acad. Sci. USA 99, 3194-3198.

Steriade, M. (2001). Impact of network activities on neuronal properties in corticothalamic systems. J. Neurophysiol. 86, 1-39.

Swanson, L. W. (1982). The projections of the ventral tegmental area and adjacent regions: a combined fluorescent retrograde tracer and immunofluorescence study in the rat. Brain Res. Bull. 9, 321-353.

Taussig, R., Iniguez-Lluhi, J. A., and Gilman, A. G. (1993). Inhibition of adenylyl cyclase by Gi alpha. Science 261, 218-221.
Treves, A., and Rolls, E. T. (1994). Computational analysis of the role of the hippocampus in memory. Hippocampus 4, 374-391.

Tucek, S., Michal, P., and Vlachova, V. (2002). Modelling the consequences of receptorG-protein promiscuity. Trends Pharmacol. Sci. 23, 171-176.

Vazdarjanova, A and Guzowski, J. F. (2004). Differences in hippocampal neuronal population responses to modifications of an environmental context: evidence for distinct, yet complementary, functions of CA3 and CA1 ensembles. J. Neurosci. 24, 6489-6496.

Vinogradova, 0. S. (2001). Hippocampus as comparator: role of the two input and two output systems of the hippocampus in selection and registration of information. Hippocampus 11, 578-598.

Witter, M. P., and Moser, E. I. (2006). Spatial representation and the architecture of the entorhinal cortex. Trends Neurosci. 29, 671-678.

Wittmann, B. C., Schott, B. H., Guderian, S., Frey, J. U., Heinze, H. J., and Duzel, E. (2005). Reward-related FMRI activation of dopaminergic midbrain is associated with enhanced hippocampus-dependent long-term memory formation. Neuron 45 , 459-467.

Wu, J., and Hablitz, J. J. (2005). Cooperative activation of D1 and D2 dopamine receptors enhances a hyperpolarization-activated inward current in layer I interneurons. $J$. Neurosci. 25, 6322-6328.

Yoshida, M., Hayashi, H., Tateno, K., and Ishizuka, S. (2002). Stochastic resonance in the hippocampal CA3-CA1 model: a possible memory recall mechanism. Neural Netw. $15,1171-1183$.

Zola-Morgan, S., and Squire, L. R. (1986). Memory impairment in monkeys following lesions limited to the hippocampus. Behav. Neurosci. 100, 155-160.

Zucker, R. S. (1973). Changes in the statistics of transmitter release during facilitation J. Physiol. 229, 787-810. 